\title{
Uso de concentrados autólogos de plaquetas como terapia regenerativa de enfermedades crónicas del aparato musculoesquelético equino
}

\author{
Use of autologous platelet concentrates as regenerative therapy for chronic \\ diseases of the equine musculoskeletal system
}

JU Carmona*, C López, CE Giraldo

Grupo de Investigación Terapia Regenerativa, Departamento de Salud Animal,

Universidad de Caldas, Manizales, Caldas, Colombia

\begin{abstract}
SUMMARY
Platelets are of pivotal importance for wound healing since they release growth factors that in turns produce chemotaxis, both cellular proliferation and differentiation, angiogenesis, and extracellular matrix deposition. The use of autologous platelet concentrates (APCs) has been proposed for accelerating wound healing, decreasing inflammation, to stimulate the regenerative capability of the injured tissues, to decrease the fibroblastic activity and to avoid the production of non functional scarring tissue. APCs could be obtained by several methods. Each method produces APCs of different both cellular and molecular quality. Recently, some basic and clinical information that justifies the usage of APCs for the treatment of degenerative musculoskeletal diseases in horses, such as osteoarthritis, tendinopathies and desmopathies has been published.
\end{abstract}

Palabras clave: equino, enfermedades musculoesqueléticas, plasma rico en plaquetas, factores de crecimiento.

Key words: equine, musculoskeletal diseases, platelet rich plasma, growth factors.

\section{INTRODUCCIÓN}

La cicatrización de heridas está dirigida por una sucesión de complejos mecanismos celulares y moleculares. Muchas células están involucradas en este proceso. Estas producen y son sensibles a una infinidad de moléculas (por ejemplo: citocinas, factores de crecimiento (GFs) y eicosanoides, entre otros) que permiten, en condiciones fisiológicas, la reparación o incluso la regeneración de los tejidos lesionados (Theoret 2005). Las plaquetas (PLTs) desempeñan un papel primordial en la cicatrización de las heridas, ya que estos fragmentos citoplásmicos además de poseer propiedades hemostáticas (Hartwig e Italiano 2003) también poseen propiedades proinflamatorias, reguladoras (Mannaioni y col 1997) y acciones regenerativas, las cuales están mediadas por la interacción con células (neutrófilos y células endoteliales) y por la liberación de GFs, quimiocinas y otras moléculas (Anitua y col 2004).

Se ha propuesto el uso de concentrados autólogos de plaquetas (APCs) en seres humanos para estimular la cicatrización de heridas en cirugía oral y maxilofacial (Marx y col 1998, Anitua 1999, Carlson y Roach 2002), en cirugía plástica (Powell y col 2001, Bhanot y Alex 2002)

Aceptado: 24.03.2010.

* Calle 65 N$^{\circ}$ 26-10, Manizales, Caldas, Colombia; carmona@ucaldas. edu.co y ortopédica (Lowery y col 1999, Sánchez y col 2003). En medicina equina los APCs han sido empleados para el tratamiento de heridas en las extremidades (Carter y col 2003) y enfermedades musculoesqueléticas crónicas (Carmona y col, 2009, b, Waselau y col 2008, Abellanet 2009). Los objetivos de esta revisión son describir la razones biológicas (fisiológicas), experimentales y clínicas que sustentan el uso de APCs en caballos con enfermedades crónicas del aparato musculoesquelético, tales como la osteoartritis, tendinopatias y desmopatías.

\section{GÉNESIS, FISIOLOGÍA Y BIOQUÍMICA DE LAS PLAQUETAS}

Los megacariocitos (MKs) son los precursores de las PLTs. Estas células se desarrollan a partir de células progenitoras mieloides multipotenciales $\mathrm{CD} 34+$ que residen en el tejido hemopoyético y el torrente sanguíneo. Los MKs representan aproximadamente el 0,1-0,5\% de las células nucleadas de la médula ósea. Estas células se encuentran en la región más profunda de los sinuosidades capilares de la médula ósea y emiten prolongaciones citoplasmáticas (proplaquetas) que están en contacto con la sangre. Estas prolongaciones son seccionadas y las PLTs son liberadas al torrente sanguíneo (Hartwig e Italiano 2003). Las plaquetas equinas son fragmentos citoplasmáticos discoides de 5-7 $\mu \mathrm{m}$ de largo y 1-3 $\mu \mathrm{m}$ de ancho (Leven 2000, Paes-Leme y col 2006); aunque es frecuente observar PLTs grandes ( $>20 \mathrm{~m} \mu)$ en el torrente sanguíneo (Argüelles y col 2006). 


\section{MEMBRANA DE LAS PLAQUETAS Y SUS RECEPTORES}

La membrana de las plaquetas se compone de tres capas: glicocalix, capa fosfolipídica y submembranosa (Tablin 2000). El glicocalix es la capa exterior que contiene receptores glicoproteicos implicados en la activación y adhesión de las PLTs. Estas glicoproteínas constituyen los antígenos de membrana de las PLTs, que se dividen en tres familias: integrinas, proteínas ricas en leucina y selectinas. Una bicapa fosfolipídica asimétrica con propiedades anticoagulantes constituye la capa central. La estructura de esta capa es idéntica a la de otras células con dominios de proteínas periféricas y transmembranales que actúan como receptores de membrana (Tablin 2000). La capa submembranosa contiene los microtúbulos de actina y actúa como el esqueleto que da la forma discal a la plaqueta en reposo. Esta capa interviene activamente en los procesos de señalización plaquetaria (Spencer y Becker 1997).

Las integrinas producen agregación y adhesión plaquetaria (Gentry 2000, Tablin 2000, Pelagalli y col 2003). Las integrinas están constituidas por dos subunidades, $\alpha$ y $\beta$, las cuales no están ligadas covalentemente. Las integrinas están conectadas de forma interna con el citoplasma de las PLTs por una sola cola y externamente con el medio por varias subunidades con dominios extracelulares. En la parte interior las integrinas están asociadas con las proteínas de señalización (proteínas G, tirosina-quinasas) $\mathrm{y}$ fosfoinositoles.

La $P$-selectina es la principal molécula de adhesión de las PLTs y MKs. Esta glicoproteína está presente en la superficie de los gránulos $\alpha$ que interactúan con fibrinógeno, factor von Willebrand (vWF), fibronectina y vitronectina (Mannaioni y col 1997, Gentry 2000, Pelagalli y col 2003). La externalización de la integrina $P$-selectina está relacionada con la activación de las PLTs equinas (Segura y col 2006).

\section{CITOPLASMA Y GRÁNULOS DE LAS PLAQUETAS}

La red citoplásmica de las PLTs está constituida por dos tipos de actina (globular y filamentosa). La actina filamentosa actúa como soporte estructural para diferentes gránulos plaquetarios y mitocondrias. La respuesta plaquetaria se produce por una actividad contráctil mediada por la polimerización del complejo actina-miosina. Los microtúbulos citoplasmáticos mantienen la forma discoide de las PLTs y dirigen los movimientos generados por actina-miosina (Gentry 2000, Hartwig e Italiano 2003). Las PLTs de los mamíferos contienen tres tipos de gránulos: lisosomales, densos y alfa $(\alpha)$ (Mannaioni y col 1997, Pelagalli y col 2002). Los gránulos lisosomales contienen hidrolasas ácidas, guanina, fosfolipasas y quinasas, que actúan como enzimas proteolíticas e hidrolíticas (Tablin 2000). Los gránulos densos almacenan ATP, ADP, calcio, fósforo y serotonina (figura 1A). El ADP induce la

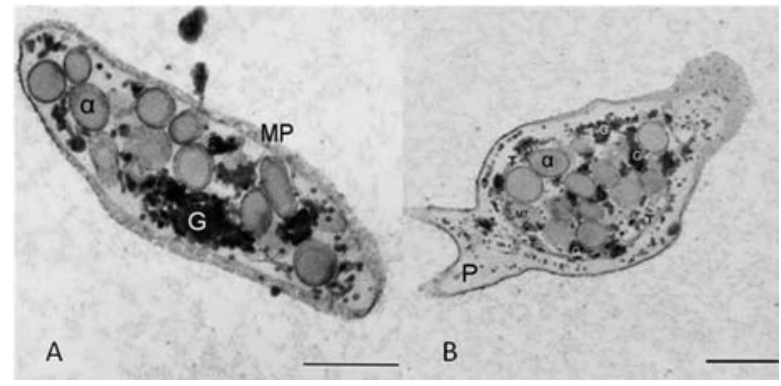

Figura 1. Micrografías obtenidas mediante microscopía electrónica de transmisión (MET) de una plaqueta equina normal (A) y de una plaqueta equina activada. MP: membrana plasmática. G: glicógeno. $\alpha$ : gránulos alfa. P: pseudópodo. MT: mitocondria. T: microtúbulos. La barra representa $1 \mu \mathrm{m}$ (Cortesía de la Pfra. Fabiola Paes Leme).

Micrographies obtained by transmission electron microscopy (TEM) of a normal equine platelet (A) and an activated equine platelet (B). MP: plasmatic membrane. G: glycogen. $\alpha$ : alpha granules. P: pseudopodium. MT: mitocondria. T microtubules. The bar represents $1 \mu \mathrm{m}$ (Courtesy by Pfr. Fabiola Paes Leme).

migración plaquetaria y en combinación con la serotonina produce la contracción de las arterias lesionadas. El ATP antagoniza la acción del ADP (Pelagalli y col 2002). Los gránulos $\alpha$ contienen varias moléculas (citocinas, quimiocinas, GFs, entre otras), algunas específicas para las PLTs (por ejemplo: factor plaquetario 4 y $\beta$-tromboglobulina) y otras que no son específicas para ellas, tales como la albúmina, condroitín 4-sulfato, fibrinógeno, fibronectina, trombospondina, factor $\mathrm{V}$, factor Va y factor von Willebrand (Mannaioni y col 1997, Anitua y col 2004). Estas proteínas son importantes para todas las funciones plaquetarias, tales como la formación y crecimiento de trombos, modulación inflamatoria y la síntesis de matriz extracelular (ECM) durante la cicatrización de heridas (Gentry 2000). Los gránulos $\alpha$ almacenan principalmente siete GFs directamente implicados en la cicatrización de las heridas, entre ellos: factor de crecimiento derivado de las plaquetas (PDGF), factor de crecimiento transformante beta 1 (TGF- $\left.\beta_{1}\right)$, TGF- $\beta_{2}$, factor de crecimiento epidérmico (EGF), factor de crecimiento endotelial vascular (VEGF), factor de crecimiento insulínico tipo 1 (IGF-I) y factor de crecimiento de hepatocitos (HGF) (Anitua y col 2005, Weibrich y col 2005). El cuadro 1 muestra un resumen que describe las principales características y la acción biológica de estas proteínas durante la cicatrización de heridas.

\section{RESPUESTA PLAQUETARIA}

Cuando se produce lesión tisular, se dispara una fuerte interacción celular. Se producen respuestas plaquetarias independientes, tales como el cambio de forma, transformación interna, secreción de gránulos (figura 1B) (Paes-Leme y col 2006), formación del tapón hemostático 
Cuadro 1. Algunos factores de crecimiento y quimiocinas contenidas en los gránulos $\alpha$ de las plaquetas Some growth factors and chemokines contained in platelet $\alpha$-granules

Factor de crecimiento/Quimiocinas y sus formas biológicas y receptores

Factor de crecimiento derivado de las plaquetas (PDGF). PDGF-AA, -BB, -AB, -CC y -DD. Dos receptores (PDGFRs): $\alpha$ y $\beta$

Factor de crecimiento transformante beta (TGF- $\beta$ ). TGF- $\beta 1,-\beta 2$, $-\beta 3$. Cinco receptores (T $\beta$ Rs). T $\beta$ RI y T $\beta$ RII son los más importantes

Factor de crecimiento epidérmico (EGF). Un receptor EGF (EGFR)

Factor de crecimiento vascular endotelial (VEGF). VEGF-B, -C, -D y dos proteínas VEGF. Dos receptores: VEGFR-1 y R-2

Factor de crecimiento de fibroblastos (FGF). FGF-1, -2, -4, -7, -9, -10 y -19.

Factor de crecimiento insulínico (IGF). IGF-I y IGF II. Dos receptores: IGF-I-R y - II-R Factor de crecimiento de hepatocitos (HGF). Proteína de unión HGF (HGF-BP)

Factor plaquetario 4 (PF-4). Condroitin sulfato (receptor)

Betatromboglobulina ( $\beta$-TG). Tres péptidos relacionados
Efectos biológicos durante la cicatrización de las heridas

Este es un quimiotáctico e inductor de proliferación celular. Estimula la angiogénesis y promueve la expresión de las metaloproteínas de matriz (MMP)-1 y su inhibidor tisular (TIMP-1) en la última fase de la remodelación. Los productos de la proliferación de fibroblastos, migración epitelial, vascularización extensiva y la infiltración de neutrófilos.

Regula la expresión de colágenos y fibronectina. Restringe la degradación de la matriz extracelular (ECM). Disminuye la expresión de las MMPs, promueve la síntesis de TIMPs y del factor de crecimiento de fibroblastos y la producción de angiogenésis. TGF- $\beta 3$ tiene efectos antifibróticos. Interacción con T $\beta R I$ produce proliferación celular y con T $\beta$ RII síntesis de ECM.

Induce la proliferación celular, diferenciación y motilidad. Es altamente expresado en el margen de las heridas, promueve la reepitelización. EGF induce la expresión de MMP-1 y regula el cambio de colágeno tipo I.

Promueve la vascularización de los tejidos lesionados y facilita el arribo de células inflamatorias y reparadoras. Tiene efectos sobre la proliferación de células del endotelio vascular.

FGF-1 (FGF básico) es una potente proteína angiogénica y controla los depósitos de ECM, puesto que inhibe la síntesis del colágeno tipo I.

IGF es un péptido anabólico. Produce proliferación celular y depósito de ECM.

Este péptido tiene efectos angiogénicos, puesto que incrementa la expresión de VEGF.

Produce quimiotaxis de leucocitos y la adhesión de neutrófilos a las células endoteliales. Es un péptido antiangiogénico, puesto que interactúa directamente con FGF o VEGF mediante el bloqueo de sus receptores de superficie celular. Inhibe la apoptosis de monocitos e induce la diferenciación de estas células a macrófagos

Estas quimiocinas producen quimiotaxis de neutrófilos y degranulación plaquetaria. En el último estado de inflamación, $\beta$-TGs desensibilizan la degranulación de neutrófilos y actúan como proteínas antiinflamatorias.
Referencias

Nimmi 1997, Reigstad y col 2005, Theoret 2005

Braun y col 2002, Todorovic y col 2005, Huang y Huang 2005

Nimmi 1997, Calvin 1998

Nimmi 1997, Ferrara 2001, Braun y col 2002, Theoret 2005

Nimmi 1997, Braun y col 2002, Theoret 2005

Harridge 2003, Frisbie y col 2000

Catlow 2003, Anitua y col 2005

Mannaioni y col 1997, Boehlen y Clemetson 2001

Mannaioni y col 1997, Boehlen y Clemetson 2001 primario y retracción del coágulo (Gentry 2000, Tablin 2000). Moléculas de la superficie plaquetaria, como las integrinas, regulan la capacidad de comunicación intercelular durante la formación del tapón hemostático, la respuesta inflamatoria y los procesos de reparación de tejidos (Mannaioni y col 1997). La expresión cinética es diferente para cada tipo de gránulo plaquetario. Primero, los gránulos $\alpha$ liberan sus contenidos como consecuencia de estímulos de baja intensidad. Luego, los gránulos densos son activados y, finalmente, los gránulos lisosomales liberan sus productos proteolíticos (Gentry 2000, Pelagalli y col 2002). Esta cadena de eventos se conoce 
como "reacción de liberación plaquetaria" (Pelagalli y col 2003). Las plaquetas equinas son especialmente sensibles al ADP, colágeno y factor activador de plaquetas (Pelagalli 2003). Las plaquetas y sus proteínas secretadas, principalmente GFs y quimiocinas, disparan el inicio del proceso inflamatorio y secuencialmente coordinan el incremento o la disminución del efecto de muchas células y moléculas implicadas en la cicatrización de las heridas (cuadro 2). Estos procesos aún no son bien comprendidos.

Marx (2004) señaló que la clave del efecto regenerativo de los APCs (acuñado por él como plasma rico en plaquetas -PRP_) está asociada con el uso de "plaquetas vivas". En ese sentido, las plaquetas deberían ser manipuladas con delicadeza durante la extracción venosa y subsecuentemente durante la preparación e inyección del APC. La inyección de una dosis superior a lo normal de “plaquetas vivas” en tejidos lesionados podría dar lugar a una respuesta similar a la inducida por estos fragmentos citoplasmáticos cuando ocurre una lesión tisular. Aunque Marx (2004) declaró que el número de PLTs concentradas en un PRP debería ser mayor que 1 millón de fragmentos citoplasmáticos por $\mu \mathrm{L}$, los autores de esta revisión y otros como Anitua y col (2004) han observado que concentraciones hasta de 300.000 PLTs por $\mu \mathrm{L}$ pueden inducir un efecto terapéutico similar.

Cuadro 2. Patobiología de la cicatrización de heridas: células, citocinas, factores de crecimiento y otras moléculas implicadas en este proceso.

Pathobiology of wound healing: cells, cytokines, growth factors and other molecules implicated in this process.
Fases de la cicatrización
Principales características y células implicadas
Factores de crecimiento implicados y otras moléculas

Diseñada para proteger el cuerpo contra la pérdida
excesiva de sangre y la invasión de sustancias extrañas.
Células implicadas: plaquetas, células endoteliales,
neutrófilos y monocitos.

Proliferativa

(fibroplasia)

Angiogenésis

Epitelización

Contracción

Depósito y remodelación de matriz

Comienza la formación de tejido de granulación necesario para la migración celular y la deposición de colágeno. Células implicadas: macrófagos, fibroblastos y queratinocitos.

Este proceso se superpone con fibroplasia. Es necesario para transportar el oxígeno y nutrientes al tejido de granulación. Comienza con la ruptura de la membrana basal por la acción de colagenasas y plasminógeno activador secretado por las células endoteliales. Células implicadas: las células endoteliales y pericitos.

Es la fase más lenta del proceso de cicatrización de heridas. Comienza 1 a 2 días después de producidas las heridas y hay una reconstitución de las células de la epidermis y otros epitelios. Se produce una migración de los queratinocitos desde el borde del corte de la epidermis y a través del defecto, posiblemente gracias a la disolución temporal de desmosomas y hemidesmosomas. La migración se produce gracias a la formación de pseudópodos.

La contracción de la herida determina la velocidad de cicatrización por segunda intención. El día 7 después de producida la herida, los defectos de la piel se reducen gracias al movimiento centrípeto de la piel intacta circundante.

La fase de maduración se caracteriza por la disminución del número de fibroblastos y alcanzar el equilibrio entre la producción de colágeno y su lisis. El equilibrio entre la síntesis de colágeno y la degradación durante la fase de remodelación depende de la presencia simultánea de metaloproteinasas de matriz (MMPs) y sus inhibidores naturales específicos de tejidos (TIMPs).
Factores de coagulación, fibrina, fibronectina, trombospondina, bradiquinina, $\mathrm{C} 3 \mathrm{a} / \mathrm{C} 5 \mathrm{a}$, histamina, leucotrienos, P-selectina, IL- $1 \beta$, TNF- $\alpha$, PDGF, TGF- $\alpha$, TGF- $\beta$, EGF, IGF, VEGF, HGF, PF-4, $\beta$-TG.

EGF, TGF- $\alpha$, TGF- $\beta$, FGF-1, HB-EGF, PDGF, IL-6, VEGF, IL-4, C5a, colágeno tipo I y III fragmentos y fibronectina.

FGF, TNF- $\alpha$, IL- 8 , ácido láctico, aminas biogénicas, TGF- $\beta$, VEGF y PDGF

EGF, factor de crecimiento de los queratinocitos (KGF), TGF- $\beta$

Fibronectina y receptores de integrina $\beta_{1}$, PDGF y interferón gamma (IFN- $\gamma$ )

MMPs

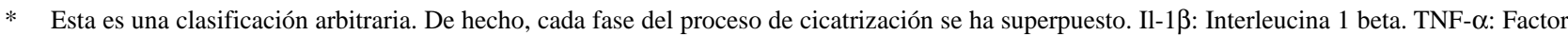
de Necrosis Tumoral alfa. C: Complemento. Otras abreviaturas, tal como en el cuadro 1. 


\section{MÉTODOS UTILIZADOS PARA LA PREPARACIÓN DE CONCENTRADOS AUTÓLOGOS DE PLAQUETAS}

Whitman y col (1997) introdujeron el uso de APCs (gel autólogo de plaquetas) en cirugía maxilofacial humana para aumentar el piso de los senos maxilares como un sustitutivo del pegamento de fibrina autóloga (Matras 1982). Después de esto, muchos dispositivos automatizados, semiautomatizados y métodos manuales se han desarrollado para la concentración de plaquetas en seres humanos (Zimmermann y col 2001, 2003, Appel y col 2002, Weibrich y col 2002, $2003^{\mathrm{a}}$, b Eppley y col 2004). Estos dispositivos concentran más PLTs, leucocitos y GFs, que algunos procedimientos de aféresis (Marx y col 1998) y el método del tubo (Weibrich y col 2005, Tamimi y col 2007).

Todas las técnicas utilizadas para preparar APCs presentan ventajas e inconvenientes. Aún no se ha desarrollado un método o dispositivo ideal para concentrar plaquetas $\mathrm{y}$ factores de crecimiento. El sistema de aféresis requiere alta tecnología y personal experimentado y un gran volumen de sangre (> $450 \mathrm{~mL}$ ) (Marx y col 1998, Weibrich y col 2002 ${ }^{\mathrm{a}}$ ). Sin embargo, se presenta un bajo riesgo de contaminación bacteriana durante la preparación de los APCs (Vasconcelos y col 2003).

Los sistemas semiatomatizados (buffy coat) permiten concentrar un elevado número de PLTs y GFs en comparación con las otras dos técnicas (Zimmermann y col 2001, Appel y col 2002, Weibrich y col 2002 , $2003^{\mathrm{a}, \mathrm{b}}$, Zimmermann y col 2003, Eppley y col 2004) y, además, el riesgo de contaminación bacteriana es menor (Vasconcelos y col 2003) que con el método manual (tubo) (Weibrich y col 2005). Sin embargo, estos dispositivos también concentran un elevado número de leucocitos y son costosos. Es importante señalar que la función exacta de los leucocitos en los APCs no se ha establecido. Sin embargo, se cree que la concentración de un gran número de glóbulos blancos en los APCs podría ser perjudicial para los tejidos tratados (Zimmermann y col 2003).

Los métodos manuales (tubo) son sencillos y baratos; sin embargo, requieren de un estricto manejo aséptico para evitar la contaminación bacteriana (Weibrich y col 2005, Tamimi y col 2007, Álvarez y col 2010). Las ventajas del método del tubo incluyen su simplicidad y bajo costo, mientras que los métodos semiautomatizados son costo-limitantes, ya que requieren kits y centrífugas especializadas.

Los tres métodos generales utilizados para la obtención de APCs en seres humanos han sido validados en caballos. Se ha evaluado la capacidad para concentrar PLTs, leucocitos, algunos GFs (Carter y col 2003, Sutter y col 2004, Argüelles y col 2006, Schnabel y col 2007, Carmona y col 2008) y óxido nítrico (Carmona y col 2008) (cuadro 3).

\section{INVESTIGACIÓN BÁSICA QUE SUSTENTA EL USO CLÍNICO DE CONCENTRADOS AUTÓLOGOS DE PLAQUETAS EN CABALLOS}

Las plaquetas contienen GFs que han sido evaluados individualmente en cartílago y tendón equino. De estos, el PDGF-BB e IGF-I han demostrado efectos positivos

Cuadro 3. Técnicas utilizadas para la preparación de concentrados de plaquetas autólogos equinos. Comparación de algunos aspectos celulares y moleculares.

Techniques used for preparing equine autologous platelet concentrates. Comparison of some cellular and molecular aspects.

\begin{tabular}{|c|c|c|c|c|c|c|c|c|c|c|}
\hline Métodos & $\begin{array}{c}\text { Plaquetas } \\
\mathrm{x} 10^{3} / \mu \mathrm{l}\end{array}$ & $\begin{array}{l}\text { WBCs } \\
\times 10^{3} / \mu 1\end{array}$ & $\begin{array}{l}\mathrm{TGF} \beta_{1} \\
\mathrm{ng} / \mathrm{ml}\end{array}$ & $\begin{array}{l}\mathrm{TGF} \beta_{2} \\
\mathrm{ng} / \mathrm{ml}\end{array}$ & $\begin{array}{l}\mathrm{TGF} \beta_{3} \\
\mathrm{pg} / \mathrm{ml}\end{array}$ & $\begin{array}{c}\text { PDGF-AB } \\
\mathrm{ng} / \mathrm{ml}\end{array}$ & $\begin{array}{l}\text { PDGF-BB } \\
\mathrm{ng} / \mathrm{ml}\end{array}$ & $\begin{array}{l}\text { IGF-I } \\
\mathrm{ng} / \mathrm{ml}\end{array}$ & $\begin{array}{l}\text { Oxido nítrico } \\
\qquad(\mu \mathrm{M})\end{array}$ & Referencias \\
\hline $\begin{array}{l}\text { Secquire PRP } \\
\text { System }\end{array}$ & 1.472 & 32,5 & 15,3 & 1 & - & - & - & 107,4 & - & Sutter y col 2004 \\
\hline $\begin{array}{l}\text { Smart PReP } 2 \\
\text { system }\end{array}$ & 395 & 4,48 & $\sim 9,5$ & - & - & - & $\sim 8,5$ & $\sim 200$ & - & Schnabel y col 2007 \\
\hline Aféresis & $490-55$ & 33,7 & $\begin{array}{c}7 \\
, 4-23,6\end{array}$ & 4,3 & - & 7,4 & - & 183,4 & - & $\begin{array}{l}\text { Carter y col } 2003, \\
\text { Sutter y col } 2004\end{array}$ \\
\hline $\begin{array}{l}\text { Aféresis más } \\
\text { filtración }\end{array}$ & 2.172 & 61,2 & 57,9 & - & - & - & - & - & - & Sutter y col 2004 \\
\hline $\begin{array}{l}\text { Método del } \\
\text { tubo }\end{array}$ & 272 & 8,4 & 10,5 & - & 30.772 & - & - & - & 35 & $\begin{array}{l}\text { Argüelles y col 2006, } \\
\text { Carmona y col } 2008\end{array}$ \\
\hline Sangre entera & $158-165$ & 6,371 & $5,58,3$ & 1,2 & $<30.774$ & - & $\sim 3$ & 171,5 & 33 & $\begin{array}{l}\text { Sutter y col } 2004, \\
\text { Argüelles y col 2006, } \\
\text { Carmona y col } 2008\end{array}$ \\
\hline
\end{tabular}

WBCs: Leucocitos. Otras abreviaturas, tal como en el cuadro 1. 
en tendón equino in vitro (Haupt y col 2006) e in vivo (Dahlgren y col 2002). Lo mismo ha sido documentado en condrocitos equinos in vitro, especialmente con IGF-I (Frisbie y col 2000) y TGF- $\beta_{1}$ (Fortier y col 1997). Estos GFs incrementan la síntesis de ECM del cartílago articular y producen proliferación de condrocitos.

La investigación básica del efecto de los APCs en caballos se ha centrado en estudios en sistemas in vitro (Smith y col 2006, Schnabel y col 2007, 2008, McCarrel y Fortier 2009) y en modelos de tendinitis (Maia y col 2009, Bosch y col 2010). Se ha dado gran preponderancia al efecto de los APCs sobre el metabolismo de tenocitos y/o miofibroblastos y la matriz extracelular de tendón y/o ligamento suspensorio de caballos. Sin embargo, hasta el momento (según lo revisado por los autores), no se ha descrito el efecto in vitro de los APCs sobre cartílago, menisco o membrana sinovial de equinos. Los APCs aumentan la capacidad metabólica y proliferativa de tenocitos y fibroblastos, la cual se hace evidente al incrementar la expresión de colágeno tipo I y la síntesis de proteína oligomérica de matriz del cartílago (COMP) (Smith y col 2006, Schnabel y col 2007, 2008). Por otra parte, Anitua y col (2005) demostraron in vitro, en explantes de tendón humano, que estas sustancias promovían la angiogénesis mediante el incremento de la expresión del factor de crecimiento vásculo-endotelial (VEGF) y el factor de crecimiento hepatocitario (HGF).

Recientemente, se ha publicado una investigación sobre el efecto de un APC (obtenido mediante el método del tubo) en un modelo equino de tendinitis inducida por colagenasa (Maia y col 2009) y otra sobre el efecto de un APC (obtenido mediante un método semiautomatizado) sobre la calidad de la reparación de lesiones tendinosas centrales (core lesion) inducidas mecánicamente en caballos. En el primer estudio se observó que a los 36 días los caballos tratados con APC tenían una mejor disposición histológica de las fibras de colágeno y fibroblastos sobre la matriz de sus tendones en comparación con los caballos del grupo control. Sin embargo, el número de fibroblastos y vasos sanguíneos no fue diferente entre ambos grupos (Maia y col 2009). En el segundo estudio se observó que a los 6 meses el grupo de caballos tratados con APC presentó una mejor arquitectura histológica, mayor contenido de colágeno, glicosaminoglicanos, DNA y mayor resistencia ténsil que el grupo control (Bosch y col 2010). Se puede apreciar que ambos métodos para concentrar plaquetas produjeron resultados biológicos similares en esos estudios experimentales.

En cuanto al efecto in vitro de los APCs sobre el metabolismo de tejidos articulares, en porcinos se ha observado que producen proliferación de condrocitos y aumento de la síntesis de su matriz extracelular (Akeda y col 2006) y en sinoviocitos de personas con osteoartritis promueven la síntesis de ácido hialurónico y la expresión de VEGF (Anitua y col 2007).

\section{USO CLÍNICO DE CONCENTRADOS AUTÓLOGOS DE PLAQUETAS COMO TRATAMIENTO DE ENFERMEDADES DEGENERATIVAS DEL APARATO LOCOMOTOR EQUINO}

La historia del uso clínico de los APCs como tratamiento de enfermedades degenerativas del aparato musculoesquelético de caballos, tales como tendinopatía (principalmente) del tendón flexor digital, desmopatía del ligamento suspensor y osteoartritis es muy reciente. En Estados Unidos existe predilección por el uso de dispositivos semiautomatizados, tales como el Secquire (PPAI Medical, Fort Myers, FL, USA) (Waselau y col 2008) y el SmartPReP2 system (Harvest Technologies, Plymouth, MA, USA), para preparar APCs equinos con finalidades terapéuticas (Schnabel y col 2007). Entretanto, en Europa continental y en algunos países de Latinoamérica se emplea el método manual del tubo (Argüelles y col 2006, Carmona y col 2008). Un aspecto importante que se debe considerar es que independientemente del método empleado (tal como se ha observado recientemente en estudios experimentales (Maia y col 2009, Bosch y col 2010)), se han obtenido buenos resultados en caballos con diferentes enfermedades locomotoras. Sin embargo, el método del tubo tiene la ventaja de ser muy barato y efectivo en comparación con los métodos semiautomatizados (Monteiro y col 2009).

\section{TRATAMIENTO DE LESIONES DEGENERATIVAS DE TENDONES Y LIGAMENTOS}

Se ha descrito el uso de APCs en casos de tendinopatía (Abellanet 2009, Carmona y col 2009ª) y desmopatía del ligamento suspensorio (Waselau y col 2008, Abellanet 2009, Carmona y col 2009a). Los APCs (obtenidos mediante el método del tubo) fueron utilizados inicialmente en un pequeño número de caballos con afecciones tendinosas y ligamentosas (Carmona y col 2009a). Los pacientes de ese estudio presentaron una mejoría clínica y ecográfica, pero esa investigación incluyó un bajo número de pacientes $(\mathrm{n}=5)$ y no había un grupo control. Sin embargo, el tratamiento fue seguro, ya que las inyecciones de APCs no indujeron reacciones adversas en los caballos tratados. Waselau y col (2008) describieron los resultados de una inyección de un APC (obtenido mediante método semiautomatizado) seguido de un programa de ejercicio controlado como tratamiento de desmitis moderada o severa del cuerpo del ligamento suspensorio en 9 caballos de carreras. Los pacientes alcanzaron su nivel atlético entre $6,5-17$ meses poslesión y corrieron consecutivamente durante dos años. Sin embargo, en ese estudio no había grupo control.

Recientemente, Abellanet (2009) describió los resultados de un estudio clínico controlado en el que se evaluó el uso de APCs (obtenidos mediante el método del tubo (Argüelles y col 2006, Carmona y col 2008, 2009)) en 72 
caballos con tendinopatía del tendón flexor digital superficial, 10 con tendinopatía del flexor digital profundo y 16 con lesiones del ligamento suspensorio. Los caballos con tendinopatía del tendón flexor digital superficial presentaron una mejoría clínica del $80 \%$ vs $45 \%$ para el grupo control $(n=9)$. De los caballos que fueron tratados con APCs presentaron recidiva el $22 \%$ en comparación con el $80 \%$ del grupo control. Los caballos con tendinopatía del flexor digital profundo que recibieron tratamiento con APCs presentaron mejoría del $100 \%$ en comparación con el $0 \%$ del grupo control $(\mathrm{n}=4)$ y una recidiva del $17 \%$ en los caballos tratados. Los caballos con desmopatía del ligamento suspensor presentaron mejoría clínica el $90 \%$ vs $0 \%$ del grupo control $(\mathrm{n}=16)$. De los caballos que fueron tratados con APCs recayó el 10\%. En general todos los caballos tratados con APCs se recuperaron en un periodo de 6 meses.

\section{TRATAMIENTO DE OSTEOARTRITIS}

Carmona y col $\left(2009^{b}\right)$ describieron el tratamiento con APCs de 4 caballos con OA de diferentes articulaciones. Los resultados obtenidos fueron favorables ya que los APCs disminuyeron el grado de cojera y efusión sinovial por más de 8 meses. Sin embargo, en ese estudio no había un grupo control, por lo que los autores sólo pudieron concluir que la inyección intraarticular de APCs en caballos con OA era segura. Sin embargo, recientemente Abellanet (2009) evaluó el efecto clínico de la inyección intraarticular de APCs en 30 caballos con OA. En ese trabajo se observó mejoría clínica en el $75 \%$ de los caballos tratados contra $0 \%$ en el grupo de 12 caballos con OA que no recibió tratamiento. El porcentaje de recidiva en esa investigación fue del $30 \%$. Los caballos que recayeron presentaban alteraciones radiológicas severas, principalmente fragmentos óseos libres mayores de 4 mm (Abellanet 2009).

\section{EFECTOS ADVERSOS ASOCIADOS CON LA INYECCIÓN DE CONCENTRADOS AUTÓLOGOS DE PLAQUETAS}

Hasta la fecha no hay informes sobre efectos adversos secundarios derivados del uso de APCs en pacientes equinos. Los autores han realizado más de 1.000 inyecciones de APCs, obtenidos mediante el método del tubo (Argüelles y col 2006, Carmona y col 2008), sin ningún tipo de complicación, aunque algunos caballos afectados por enfermedad articular desarrollaron un ligero derrame sinovial durante las primeras 48 horas después de la inyección de APCs (Carmona y col 2009 ${ }^{\text {b }}$. Es posible que estas efusiones sinoviales transitorias estén relacionadas con la presencia de leucocitos en los APCs. Sin embargo, el PDGF liberado durante la activación plaquetaria también podría contribuir con este problema, ya que es un poderoso factor de crecimiento quimiotáctico para leucocitos periféricos (Nimmi 1997). Existe alguna desconfianza entre veterinarios para inyectar APCs vía intraarticular en caballos con OA, pues se piensa en la posibilidad de desarrollar una artritis séptica posinyección. Recientemente, Álvarez y col (2010) demostraron que los APCs obtenidos mediante el método del tubo no sufren contaminación bacteriana, siempre y cuando se realice un adecuado protocolo de desinfección de la piel del sitio de la venopunción y que estos sean preparados en un cuarto cerrado y sin corrientes de aire. Por otra parte, es necesario considerar que los APCs tienen una potente acción antimicrobiana contra las bacterias de la piel de los caballos (Álvarez y col, datos sin publicar), tales como Staphylococcus aureus (Bielecki y col 2007).

\section{NÚMERO Y VIABILIDAD DE PLAQUETAS, NÚMERO DE DOSIS, INTERVALO POSOLÓGICO, MOMENTO IDEAL DE APLICACIÓN VERSUS EFECTIVIDAD CLÍNICA DE LOS CONCENTRADOS AUTÓLOGOS DE PLAQUETAS}

Según la literatura revisada y particularmente por los resultados obtenidos in vivo en modelos experimentales (Maia y col 2009, Bosch y col 2010) y en estudios clínicos (Waselaw y col 2008, Abellanet 2009, Carmona y col 2009a, b) existe una buena respuesta terapéutica de los APCs a partir de dosis de plaquetas tan bajas como $300.000 / \mu \mathrm{L}$ (Maia y col 2008, Carmona y col 2009a, b, Abellanet 2009) y tan altas como más de un millón/ $\mu \mathrm{L}$ (Waselaw y col 2008). Para los autores de esta revisión la fracción celular extraplaquetaria y la viabilidad plaquetaria son aspectos más importantes que concentrar una gran cantidad de plaquetas "no viables" y de factores de crecimiento (McCarrel y Fortier 2009). Aunque existe una corriente norteamericana que recomienda congelar APCs para un posterior uso en el paciente, nuestro equipo cree que estos concentrados se deben activar y aplicar inmediatamente. Esto porque es posible que la viabilidad celular del APC y su actividad metabólica produzcan una reacción de liberación más adecuada y temporalmente sostenida de los gránulos alfa y una mejor interacción celular (Anitua y col 2004).

Los resultados de Abellanet (2009) confirman, especialmente para lesiones de tejidos blandos, que entre mayor sea el número de aplicaciones de APC/lesión, se presentará una mejor respuesta clínica y un menor número de recaídas. Los autores consideran que tres e incluso cuatro aplicación de APCs, con un intervalo de 10-15 días entre aplicación, es un esquema posológico adecuado. Este esquema terapéutico se ha basado en la impresión clínica de los autores y, por otra parte, los estudios de Abellanet (2009) indican que algunos biomarcadores anabólicos declinan 15 días después de la aplicación intraarticular o intrasinovial de APCs en caballos. Los autores consideran que los APCs pueden ser aplicados en cualquier momento de evolución de las lesiones. No se debe olvidar que 
la mayoría de las enfermedades del aparato locomotor equino son "crónicas por naturaleza" y que los episodios agudos son solamente agravamientos momentáneos de las mismas patologías (Carmona y Prades 2009). Los APCs constituyen un excelente tratamiento analgésico y antiinflamatorio para las lesiones en "fase aguda" de tendones, ligamentos y articulaciones y sólo existen "limitaciones teóricas", que sugieren su aplicación únicamente en la fase proliferativa de la lesión, especialmente tendinosa (Schnabel y col 2007).

\section{CONCLUSIÓN}

Los APCs constituyen una opción terapéutica real para el manejo de las enfermedades crónicas musculoesqueléticas del caballo. Según los resultados obtenidos en modelos experimentales y en pacientes con enfermedad natural, existe una buena respuesta terapéutica de los APCs a partir de dosis de plaquetas tan bajas como $300.000 / \mu \mathrm{L}$. Los APCs deberían ser aplicados al menos tres veces con un intervalo de 2 semanas entre aplicación. El método del tubo representa una opción simple y económica para preparar concentrados autólogos de "plaquetas viables".

Es necesario ampliar el conocimiento sobre los efectos moleculares de los APCs sobre el cartílago articular equino y realizar más estudios clínicos doble ciego que incluyan un gran número de pacientes equinos con diferentes enfermedades musculoesqueléticas y hacer estudios sobre el comportamiento de biomarcadores anabólicos y catabólicos a largo tiempo ( 3 años). Los resultados de estudios de tal magnitud no sólo serían de beneficio para los caballos, sino que tendrían una importante aplicación traslacional en seres humanos.

\section{RESUMEN}

Las plaquetas son fundamentales para la reparación tisular de las heridas, ya que secretan factores de crecimiento, los cuales inducen quimiotaxis, proliferación y diferenciación celular, neovascularización y producción de la matriz extracelular. Se ha propuesto el uso de concentrados autólogos de plaquetas (APCs) para acelerar la cicatrización de heridas, disminuir la inflamación, estimular la capacidad regenerativa de los tejidos lesionados, disminuir la actividad fibroblástica y la producción de tejido cicatricial nofuncional. Los APCs pueden ser preparados mediante diferentes métodos. Cada método produce APCs de diferente calidad celular y molecular. Recientemente, se ha generado información básica y clínica que justifica el uso de APCs en caballos con enfermedades degenerativas del aparato musculoesquelético equino como la osteoartritis, tendinopatías y desmopatías.

\section{AGRADECIMIENTOS}

Los autores agradecen a la Profesora Fabiola de Oliveira Paes Leme, MV, MSc, PhD (Universidad Federal de Minas Gerais, Belo Horizonte, MG, Brasil), por el aporte de las fotografías ultraestructurales de las plaquetas equinas presentadas en este trabajo.

\section{REFERENCIAS}

Abellanet I. 2009. La terapia de lesiones de tejidos blandos y articulaciones con plasma rico en plaquetas en caballos de deporte: evidencia clínica y bioquímica que valida su utilización. Tesis de Doctorado, Facultad de Veterinaria, Universidad Autónoma de Barcelona, España.

Akeda K, HS An, M Okuma, M Attawia, K Miyamoto, EJ Thonar, ME Lenz, RL Sah, K Masuda. 2006. Platelet-rich plasma stimulates porcine articular chondrocyte proliferation and matrix biosynthesis. Osteoarthritis Cartilage 14, 1272-1280.

Álvarez ME, CE Giraldo, JU Carmona. 2010. Contaminación bacteriana en concentrados de plaquetas de caballos. Arch Med Vet 42, 49-56.

Álvarez ME, CE Giraldo, I Samudio, JU Carmona. 2011. Actividad bactericida in vitro de concentrados de plaquetas, plasma pobre en plaquetas y plasma de equinos contra Staphylococcus aureus resistente a la meticilina. Arch Med Vet 43, en prensa.

Anitua E. 1999. Plasma rich in growth factors: preliminary results of use in the preparation of future sites for implants. Int J Oral Maxillofac Implants 14, 529-535.

Anitua E, I Andia, B Ardanza, P Nurden, AT Nurden. 2004. Autologous platelets as a source of proteins for healing and tissue regeneration. Thromb Haemost 91, 4-15.

Anitua E, I Andia, M Sánchez, J Azofra, MM Zalduendo, M de la Fuente, P Nurden, AT Nurden. 2005. Autologous preparations rich in growth factors promote proliferation and induce VEGF and HGF production by human tendon cells in culture. J Orthop Res 23, 281-286.

Anitua E, M Sánchez, AT Nurden, MM Zalduendo, M de la Fuente, J Azofra, I Andia. 2007. Platelet-released growth factors enhance the secretion of hyaluronic acid and induce hepatocyte growth factor production by synovial fibroblasts from arthritic patients. Rheumatology (Oxford) 46, 1769-1772.

Appel TR, B Pötzsch, J von Müller, JJ Lindern, SJ Bergé, RH Reich. 2002. Comparison of three different preparations of platelet concentrates for growth factor enrichment. Clin Oral Implant Res 13, 357-362.

Argüelles D, JU Carmona, J Pastor, A Iborra, L Viñals, P Martínez, E Bach, M Prades. 2006. Evaluation of single and double centrifugation tube methods for concentrating equine platelets. Res Vet Scien 81, 237-245.

Bhanot S, JC Alex. 2002. Current applications of platelet gels in facial plastic surgery. Facial Plast Surg 18, 27-33.

Bielecki TM, TS Gazdzik, J Arendt, T Szczepanski, W Król, T Wielkoszynski. 2007. Antibacterial effect of autologous platelet gel enriched with growth factors and other active substances: an in vitro study. J Bone Joint Surg Br 89, 417-420.

Boehlen F, K Clemetson. 2001. Platelets chemokines and their receptors: what is their relevance to platelet storage an transfusion practice? Transfus Med 11, 403-417.

Bosch G, HT van Schie, MW de Groot, JA Cadby, CH van de Lest, A Barneveld, PR van Weeren. 2010. Effects of platelet-rich plasma on the quality of repair of mechanically induced core lesions in equine superficial digital flexor tendons: A placebo-controlled experimental study. J Orthop Res 28, 211-217.

Braun S, U auf dem Keller, HD Beer, M Krampert, M Müller, S Werner, C Dickson, S Werner. 2002. Growth factors in development, repair and disease. Eur J Cell Biol 81, 375-382.

Calvin M. 1998. Cutaneous wound repair. Wounds 10, 12-32.

Carlson NE, RB Roach. 2002. Platelet-rich plasma: clinical applications in dentistry. J Am Dent Assoc 133, 1383-1386.

Carmona JU, D Argüelles, M Prades. 2008. Transforming growth factor beta-3 and nitric oxide levels in four autologous platelet concentrates and plasma derived from equine blood. Arch Med Vet 40, 155-160.

Carmona JU, M Prades. 2009. Platelet concentrates to treat musculoskeletal disease in horses. VDM Verlag Dr. Müller Aktiengesellschaft \& Co. KG, Saarbrücken, Germany.

Carmona JU, M Prades, D Argüelles. 2009ª . Autologous platelet concentrates as a treatment for soft tissue musculoskeletal lesions in horses. Arch Med Vet 41, 77-82. 
Carmona JU, C López, M Prades. 2009' . Use of autologous platelet concentrates obtained by the tube method as a treatment for arthropathies in horses. Arch Med Vet 41, 175-179.

Carter CA, DG Jolly, CE Worden, DG Hendren, CJ Kane. 2003. Plateletrich plasma gel promotes differentiation and regeneration during equine wound healing. Exp Mol Pathol 74, 244-255.

Catlow K, JA Deakin, M Delehedde, DG Fernig, JT Gallagher, MS Pavão, M Lyon. 2003. Hepatocyte growth factor/scatter factor and its interaction with heparin sulphate and dermatan sulphate. Bioch Soc Transact 31, 352-353.

Dahlgren LA, MCH van der Meulen, JEA Bertram, GS Starrak, AJ Nixon. 2002. Insulin-like growth factor-I improves cellular and molecular aspects of healing in a collagenase-induced model of flexor tendinitis, J Orthop Res 20, 910-919.

Eppley BL, JE Woodell, J Higgins. 2004. Platelet quantification and growth factor analysis from platelet-rich plasma: implications for wound healing. Plast Reconst Surg 114, 1502-1508.

Ferrara N. 2001. Role of vascular endothelial growth factor in regulation of physiological angiogenesis. Am J Physiol Cell Physiol 280, C1358-C1366.

Fortier LA, AJ Nixon, HO Mohammed, G Lust. 1997. Altered biological activity of equine chondrocytes cultured in a three-dimensional fibrin matrix and supplemented with transforming growth factor beta-1. Am J Vet Res 58, 66-70.

Frisbie DD, EA Sandler, GW Trotter, CW McIlwraith. 2000. Metabolic and mitogenic activities of insulin-like growth factor- 1 in interleukin1-conditioned equine cartilage. Am J Vet Res 61, 436-441.

Gentry PA. 2000. Platelet biology. Schalman's Veterinary Hematology. Lippincott Williams and Wilkins, Philadelphia, USA, Pp 459466

Harridge SD. 2003. Ageing and local growth factors in muscle. Scand J Med Scien Sport 13, 34-39.

Hartwig J, J Italiano. 2003. The birth of the platelet. J Thromb Haemost 1, 1580-1586.

Haupt JL, BP Donnelly, AJ Nixon. 2006. Effects of platelet-derived growth factor-BB on the metabolic function and morphologic features of equine tendon in explant culture. Am J Vet Res 67, 1595-1600.

Huang SS, JS Huang. 2005. TGF- $\beta$ control of cell proliferation. J Cell Bioch 96, 447-462.

Leven ER. 2000. Megakaryocytes. Schalman's Veterinary Hematology. Lippincott Williams and Wilkins, Philadelphia, USA, Pp 443-447.

Lowery GL, S Kulkarni, AE Pennisi. 1999. Use of autologous growth factors in lumbar spinal fusion. Bone 25 (S2), 478-508.

Maia L, MV de Souza, JI Ribeiro, AC de Oliveira, GES Alves, L dos Anjos Benjamin, YFR Silva, BM Zandim, JCL Moreira. 2009. Platelet-rich plasma in the treatment of induced tendinopathy in horses: histologic evaluation. J Equine Vet Sci 29, 618-626.

Mannaioni PF, GM Bello, E Masini. 1997. Platelets and inflammation: role of platelet-derived growth factor, adhesion molecules and histamine. Inflamm Res 46, 4-18.

Marx RE, ER Carlson, RM Eichstaedt, SR Schimmele, JE Strauss, KR Georgeff. 1998. Platelet rich plasma: growth factor enhancement for bone grafts. Oral Surg Oral Med Oral Pathol Oral Radiol Endod 85, 638-646.

Marx RE. 2004. Platelet-rich plasma: evidence to support its usage. J Oral Maxillofac Surg 62, 489-496.

Matras H. 1982. The use of fibrin glue in oral and maxillofacial surgery. J Oral Maxillofac Surg 40, 617.

McCarrel T, L Fortier. 2009. Temporal growth factor release from platelet-rich plasma, trehalose lyophilized platelets, and bone marrow aspirate and their effect on tendon and ligament gene expression, J Orthop Res 27, 1033-1042.

Monteiro SO, O M Lepage, CL Theoret. 2009. Effects of platelet-rich plasma on the repair of wounds on the distal aspect of the forelimb in horses. Am J Vet Res 70, 277-282.

Nimmi ME. 1997. Polypeptide growth factors: targeted delivery systems. Biomaterials 18, 1201-1225.
Paes-Leme FO, LJ Wurzinger, AC Vasconcelos, GES Alves. 2006. Ativação de plaquetas de eqüinos com laminite induzida e tratados com ketoprofeno, fenilbutazona e flunixin meglumina. Arq Bras Med Vet Zootec 58, 149-157.

Pelagalli A, P Lombardi, D d'Angelo, R Della Morte, L Avallone, N Staiano. 2002. Species variability in platelet aggregation response to different agonists. J Comp Pathol 127, 126-132.

Pelagalli A, MA Belisario, S Tafuri, P Lombardi, D d'Angelo, L Avallone, N Staiano. 2003. Adhesive properties from different animal species. J Comp Pathol 128, 127-131.

Powell DM, E Chang, EH Farrior. 2001. Recovery from deep-plane rhytidectomy following unilateral wound treatment with autologous platelet gel: a pilot study. Arch Facial Plast Surg 3, 245-250.

Reigstad L, JE Varhaug, JR Lillehaug. 2005. Structural and functional specificities of PDGF-C and PDGF-D, the novel members of the platelet-derived growth factors family. FEBS J 272, 5723-5741.

Sánchez M, J Azofra, E Anitua, I Andía, S Padilla, J Santisteban, I Mujika. 2003. Plasma rich in growth factors to treat an articular cartilage avulsion: a case report. Med Scien Sports Exerc 35, 1648-1652.

Segura D, L Monreal, S Pérez-Pujol, M Pino, A Ordinas, R Brugués, JG White, G Escolar. 2006. Assessment of platelet function in horses: ultrastructure, flow cytometry and perfusion techniques. $J$ Vet Intern Med 20, 581-588.

Schnabel LV, HO Mohammed, BJ Miller, WG McDermott, MS Jacobson, KS Santangelo, LA Fortier. 2007. Platelet rich plasma (PRP) enhances anabolic gene expression patterns in flexor digitorum superficialis tendons. J Orthop Res 25, 230-240.

Schnabel LV, HO Mohammed, MS Jacobson, LA Fortier. 2008. Effects of platelet rich plasma and acellular bone marrow on gene expression patterns and DNA content of equine suspensory ligament explant cultures. Equine Vet $J$ 40, 260-265.

Smith JJ, MW Ross, RK Smith. 2006. Anabolic effects of acellular bone marrow, platelet rich plasma, and serum on equine suspensory ligament fibroblasts in vitro. Vet Comp Orthop Traumatol 19, 43-47.

Spencer FA, RC Becker. 1997. Platelets: Structure, Function, and Their Fundamental Contribution to Hemostasis and Pathologic Thrombosis. Textbook of Coronary Thrombosis and Thrombolysis, Kluwer Academic Publishers, Norwell, USA, Pp 31-49.

Sutter WW, AJ Kaneps, AL Bertone. 2004. Comparison of hematologic values and transforming growth factor- $\beta$ and isulin-like growth factor concentrations in platelets concentrates obtained by use of buffy coat and apheresis methods from equine blood. Am J Vet Res 65, 924-930.

Tablin F. 2000. Platelet structure and function. Schalman's Veterinary Hematology. Lippincott Williams and Wilkins, Philadelphia, USA, Pp 448-452.

Tamimi FM, S Montalvo, I Tresguerres, L Blanco-Jerez. 2007. A comparative study of 2 methods for obtaining platelet-rich plasma. J Oral Maxillofac Surg 65, 1084-1093.

Theoret CL. 2005. The pathophysiology of wound repair. Vet Clin Equine 21, 1-13.

Todorovic V, V Jurukovski, Y Chen, L Fontana, B Dabovic, DB Rifkin. 2005. Latent TGF- $\beta$ binding proteins. Int J Biochem Cell Biol 37, 38-41.

Vasconcelos E, AC Figueiredo, J Seghatchian. 2003. Quality of platelet concentrates derived by platelet rich plasma, buffy coat and apheresis. Transfus Apher Sci 29, 13-16.

Waselau M, WW Sutter, RL Genovese, AL Bertone. 2008. Intralesional injection of platelet-rich plasma followed by controlled exercise for treatment of midbody suspensory ligament desmitis in Standardbred racehorses. J Am Vet Med Assoc 232, 1515-1520.

Weibrich G, RS Buch, WK Kleis, G Hafner, WE Hitzler, W Wagner. $2002^{\mathrm{a}}$. Quantification of thrombocyte growth factors in platelet concentrates produced by discontinous cell separation. Growth factors 20, 93-97.

Weibrich G, WK Kleis, G Hafner. 2002 ${ }^{\text {b }}$. Growth factor levels in the platelet-rich plasma produced by 2 different methods: curasan- 
type PRP kit versus PCCS PRP system. Int J Oral Maxillofac Implants 17, 2184-2190.

Weibrich G, WK Kleis, R Buch, WE Hitzler, G Hafner. 2003a . The Harverst Smart PReP system versus the Friadent-Schütze platelet rich plasma kit. Clin Oral Implants Res 14, 233-239.

Weibrich G, WK Kleis, G Hafner, WE Hitzler, W Wagner. $2003^{\text {b }}$. Comparison of platelet, leukocyte, and growth factor levels in point-of-care platelet-enriched plasma, prepared using a modified Curasan kit, with preparations received from a local blood bank. Clin Oral Implants Res 14, 357-362.

Weibrich G, WK Kleis, WE Hitzler, G Hafner. 2005. Comparison of the platelet concentrate collection system with the plasma-rich-ingrowth-factors kit to produce platelet rich plasma: a technical report. Int J Oral Maxillofac Implants 29, 118-123.
Whitman DH, RL Berry, DM Green. 1997. Platelet gel: an autologous alternative to fibrin glue with applications in oral and maxillofacial surgery. J Oral Maxillofac Surg 55, 1294-1299.

Zimmermann R, R Jakubietz, M Jakubietz, E Strasser, A Schlegel, J Wiltfang, R Eckstein. 2001. Different preparation methods to obtain platelet components as a source of growth factors for local application. Transfusion 41, 1217-1224.

Zimmermann R, D Arnold, E Strasser, J Ringwald, A Schlegel, J Wiltfang, R Eckstein. 2003. Sample preparation technique and white cell content influence the detectable levels of growth factors in platelet concentrates. Vox Sang 85, 283-289. 